\title{
PREFACE AND HIGHLIGHTS
}

IAU Symposium No. 156 "Developments in Astrometry and Their Impact on Astrophysics and Geodynamics" was held in Shanghai, China, from 15-19 September 1992. The symposium also commemorated the 120th anniversary of the founding of Shanghai Astronomical Observatory and the 70th anniversary of the Chinese Astronomical Society. The scientific program was presented in seven half-day sessions. All sessions were held at the Shanghai Convention Center of the Chinese Academy of Sciences. The sessions were organized around major topics such as space astrometric missions, ground-based optical techniques, very long baseline interferometric (VLBI) techniques, impact (of primarily these techniques) on astrophysics and on geodynamics, and issues related to celestial and terrestrial reference frames.

The organization of this volume is by these topics and does not necessarily reflect the order of presentations at the symposium. Approximately 100 scientific papers were presented either orally or in poster form. Most of them appear in these Proceedings as submitted by the authors in camera-ready form ready for reproduction. The editors' job was limited to follow up on a review process and to try to satisfy the most important criticisms offered by the reviewers. Major revisions, however, were not attempted sometimes sacrificing grammar, format, etc. for speedy publication. Because of the wide range of topics and the broad scope of the symposium it was also decided to forgo the customary index, assuming that entries can be located effectively through the Table of Contents. An attempt to highlight the sessions by major topics is made later in this Preface.

Five resolutions (see pp. xxviii-xxx) were adopted by the participants concerning old earth orientation data, astrometric observations, VLBI, and milli- and micro-arcsecond astrometry.

The symposium participants are indebted to the members of the Scientific Organizing Committee for their valuable guidance and assistance in planning the symposium and appreciate the support from the Local Organizing Committee in planning the local arrangements including the daily social events. The names of the SOC are given in the following pages.

The symposium is also indebted to several organizations and agencies for financial and other support and/or sponsorship. These are the International Astronomical Union and its Commissions 8, 19,24, 31, 33; the International Association of Geodesy; the National Science and Technology Committee of China; the National Natural Science Foundation of China; the Chinese Academy of Sciences; the Chinese Astronomical Society; the Shanghai Association of Science and Technology; the Shanghai Astronomical Society; and, naturally, the Shanghai Astronomical Observatory.

Returning now to the sessions, starting with the topic on SPACE OPTICAL ASTROMETRY, reduction of one year of data from the Hipparcos mission shows excellent results for more than 40,000 stars. Standard errors in position have a maximum of 1.5 mas in ecliptic latitude and 1.8 mas in longitude. Mean standard error for parallaxes is 3 mas. Unless serious hardware problems develop, parallax errors will be 1.5 mas, proper motions 1.2 and 0.8 in longitude and latitude, respectively. 
The photometric reduction shows that 85 percent of the 40,000 stars most frequently observed have standard errors of less than 0.005 magnitudes. Photometry of double stars yield components with errors of 0.01 magnitudes leading to new ways to determine stellar masses.

Observations of 60 minor planets by Hipparcos link this system to inertial reference defined by the motions of bodies in the solar system. Extension of the results obtained so far by simulation over a 2.5 year period allows one to expect a precision of 5 mas.

For a possible second Hipparcos mission, a CCD modulation detector is proposed. With a 1000 times greater light efficiency than with the present image disector tube system, it would be possible to measure all stars brighter than 14th magnitude, and with an accuracy of 0.5 mas up to 10th magnitude.

A satellite system using a mosaic of CCD detectors was also described with a projected limiting magnitude of 18 and a five-year mission, an accuracy of 0.1 mas for position and parallax, and 0.05 mas for annual proper motion at the 12th magnitude.

As far as the Hubble Space Telescope's Fine Guidance Sensor is concerned, it was shown that several mas astrometry is possible for parallaxes, proper motions, and double star observations.

During the sessions on GROUND BASED OPTICAL ASTROMETRY it was striking to learn how much activity is currently in the development of CCD meridian circles and transit instruments pushing the limiting magnitude as far as 17.5. One of the objectives is to reach the magnitude range of the bright quasars; another is the establishment of fundamental reference frames and quasi-inertial reference frames including faint stars, based entirely on ground-based observations of planetary objects, stars, and quasars.

Another interesting topic was the recognition that the rereduction of past astrometric observations with respect to the forthcoming Hipparcos frame has the potential of casting new light on earth rotation parameters determined since the beginning of the century. Since the procedures for the rereduction are somewhat laborious due to data preparation, support by IAU and other organizations/agencies appears to be necessary.

The sessions on VERY LONG BASELINE INTERFEROMETRY (VLBI) demonstrated that the VLBI technique is still the most powerful one in the determination of earth rotation parameters, nutation series, and baseline lengths. Precisions are in the range of 2-12 mm in all of these parameters, and the frequencies have been increased to the point where they can be useful in improving geodynamic models of these phenomena.

Very precise radio source catalogs have been compiled through VLBI observations. Among 700 compact extragalactic radio sources, about 300 proved to be satisfactory (precision of about 0.5 mas) to define a Celestial Reference Frame. New VLBI observatories are being developed in Hawaii, Chile, Brazil, two in Antarctica, several in Europe and Asia. The Quasat project in the former Soviet Union is also under implementation; thus the geographic distribution of VLBI stations is expected to improve considerably.

The latest data acquisition system, $\mathrm{MK} 4$, is about ready as a prototype. The recording bandwidth is expanded to about four times of the current MK3A to $1 \mathrm{Gbit} / \mathrm{s}$, and maybe even to $2 \mathrm{Gbit} / \mathrm{s}$ in the future. A new correlator is also under design.

Reports on space-based VLBI efforts indicate their potential usefulness in both astrophysics and geodynamics. Finally a few hundred microarcsecond differential VLBI technique for spacecraft tracking, a method similar to photographic astrometry but applied to radio astrometry, may open a wide range of applications for various scientific purposes such as the determination of planetary relativistic deflection.

The above observational systems, space- and ground-based optical astrometry, and VLBI, as well as other techniques such as the Global Positioning System(GPS), had a 
great IMPACT ON ASTROPHYSICS AND GEODYNAMICS. It is impossible to summarize all the accomplishments, but the most important ones presented at the symposium include the following:

- The correction to the current value of the constant of precession may be converging to the value of -0.30 "/cy, and the reason for the difference versus the current value by Fricke may be explainable.

- The combination of old photographic plates, modern high-speed accurate measuring machines, and new observational plates may permit interesting proper motion surveys.

- The new data types require the use of modern statistics as opposed to traditional methods.

- During the past year, with a core of worldwide GPS stations in continuous operation, polar motion deduced by this network (IGS) agrees at a level of 0.5 mas with that obtained by VLBI. GPS also appears to be the appropriate technique to monitor ground deformations at least on a local scale.

- A possible origin of short periodic (less than 1 year) variations of length of day (lod) may be changes in solar activity, especially at 38,50,70, and 120 days. The interface phenomena are solar wind, interplanetary magnetic field, and the atmosphere.

- Interannual fluctuations in earth rotation are related to sea level changes of the tropical Pacific. The phases would allow correlations with oceanic tides (as sea level changes) and El Niño events.

- Influences of unmodeled part of zonal tides and the atmospheric angular momentum on earth rotation have been estimated, the conclusion being that for rapid changes (shorter than 30 days) actual modeling is possible but for longer periods improvements are still needed, especially for the semiannual components.

Progress in astrophysics is severely limited by the current accuracies and resolutions of astrometric techniques. It appears that positions and proper motions of stars to 19th magnitude with an accuracy of 0.1 mas with respect to an inertial frame of reference is needed. This means that the same or better accuracy for a CELESTIAL REFERENCE FRAME has to be achieved. The symposium considered different aspects of this problem:

- The FK5 basic and the FK5 Extension catalogues are nearly in the same frame (differences are smaller than 30 mas). Comparisons between the FK5 and new observational data also show differences less than 30 mas in both declination and right ascension, except in the declination zones -75 to -90 degrees and between +80 and +90 degrees.

- Comparisons of VLA positions and those obtained by FAST on Hipparcos data give differences of about 60 mas. The difference is attributed to errors in the ground-based proper motions used to transfer the VLA positions to the current epoch.

The TERRESTRIAL REFERENCE FRAME currently is defined through the positions and motions of VLBI and artificial satellite/lunar laser observatories and is maintained by the 
International Earth Rotation Service (IERS). Connections between this frame and the individual terrestrial frames inherent in each of the above techniques as well as other geodetic frames are available through the IERS. The main limitation of the present system is in the number of observatories and their geographical distribution. The new initiative by the International Association of Geodesy (IAG) is an attempt to remedy this situation through the new International GPS Service for Geodynamics (IGS) currently under experimental development. When fully operational IGS will provide the scientific community with orbital information on all GPS satellites, earth rotation parameters of high resolution, and positions and motions for some 200 observatories well distributed over the globe. The latter should provide access to the terrestrial reference frame for most users.

As it is seen from the foregoing, the symposium covered a very large number of topics, perhaps too many for such a short meeting. The observational community was well represented, but relatively few application-oriented scientists managed to come, especially from the astrophysical community. It was for this reason that the recommendation was made that the next time around a special effort be made by the IAU to bring modern astrometrists and astrophysicists under one roof in a highly specialized symposium or colloquium.

To fit the pieces of the diverse topic mosaics into a unified picture was the responsibility of the session chairs, who fulfilled their responsibilities with exceptional skill. They were Drs. Kaj A. Strand (Space Astrometry), Hans G.Walter (Optical Techniques), Shuhua Ye (Radio Techniques), Kenneth Seidelmann (Astrophysics), Paul Pâquet (Geodynamics), Yaroslav S. Yatskiv (Celestial Reference Frames), E.Høg (Terrestrial Reference Frames), and Jean Kovalevsky (Summary Session). Suzanne Debarbat's historical comments follow this preface, which turned out to be longer than expected. 